\title{
Into the Wild: Oryza Species as Sources for Enhanced Nutrient Accumulation and Metal Tolerance in Rice
}

\author{
Felipe K. Ricachenevsky ${ }^{1 *}$ and Raul A. Sperotto ${ }^{2 *}$ \\ ${ }^{1}$ Departamento de Biologia, Programa de Pós-Graduação em Agrobiologia, Universidade Federal de Santa Maria, Santa \\ Maria, Brazil, ${ }^{2}$ Setor de Genética e Biologia Molecular do Museu de Ciências Naturais, Programa de Pós-Graduação em \\ Biotecnologia, Centro de Ciências Biológicas e da Saúde, Centro Universitário UNIVATES, Lajeado, Brazil
}

Keywords: metal tolerance, natural variation, nutrient use efficiency, rice domestication, wild rice species

\section{RICE AND Oryza GENUS DIVERSITY}

Rice (Oryza sativa L.) is one of the most important crops in the world (Elert, 2014). However, rice grain production can be affected by nutritional status, depending on the cultivation method and soil type: high levels of metals such as aluminum $(\mathrm{Al})$ and iron ( $\mathrm{Fe})$ decrease plant growth and yield, especially in acidic soils (Ricachenevsky et al., 2010; Kochian et al., 2015), while low nutrient use

OPEN ACCESS

Edited by:

Edgar Peiter,

Martin-Luther-Universität Halle-Wittenberg, Germany

Reviewed by:

Frans Maathuis,

University of York, UK

${ }^{*}$ Correspondence:

Felipe K. Ricachenevsky felipecruzalta@yahoo.com.br;

Raul A. Sperotto

rasperotto@univates.br

Specialty section: This article was submitted to Plant Nutrition,

a section of the journal Frontiers in Plant Science

Received: 23 May 2016 Accepted: 20 June 2016

Published: 29 June 2016

Citation: Ricachenevsky FK and Sperotto RA (2016) Into the Wild: Oryza Species as

Sources for Enhanced Nutrient Accumulation and Metal Tolerance in Rice. Front. Plant Sci. 7:974. doi: 10.3389/fpls.2016.00974 efficiency leads to increased fertilization needs (Koutroubas and Ntanos, 2003). Rice grains, which are key staple food for more than half of the world's population, can accumulate toxic elements such as arsenic (As), or show low concentration of key nutrients to human nutrition, such as Fe and zinc (Zn) (Sperotto et al., 2012; Ricachenevsky et al., 2015; Clemens and Ma, 2016). Thus, attempts to improve metal tolerance, nutrient use efficiency, and nutrient accumulation in grains are likely to have an impact on both agriculture and rice grains nutritional value for consumption.

Cultivated rice (O. sativa) is part of the Oryza genus, which is composed of 23 species, including the cultivated African rice Oryza glaberrima, plus 21 wild relatives (Jacquemin et al., 2013). These species show 11 different genome types (AA, BB, CC, BBCC, CCDD, EE, FF, GG, KKLL, HHJJ, HHKK; Lu et al., 2009; Jacquemin et al., 2013; Atwell et al., 2014), and have a pan-tropical distribution, growing in a broad range of environments (Atwell et al., 2014). Since O. sativa was domesticated from a limited number of O. rufipogon genotypes, its closest wild relative, it is estimated that only $10-20 \%$ of the genetic diversity found in wild species is present in cultivated rice germplasm (Zhu et al., 2007; Palmgren et al., 2014). Although many efforts were made to find natural variation within the rice germplasm that could improve nutrition-related traits in cultivated rice, the narrow genetic diversity can be a limiting factor (Li et al., 2014; Yan et al., 2016). Considering the diversity of rice wild species and their distinct growing environments (Atwell et al., 2014), we can expect that they will be adapted to different nutrient availabilities. Thus, wild relatives are a potential source of interesting alleles or even new mechanisms of metal and metalloid accumulation control. However, these genetic resources are almost unexplored, with very few studies screening these species for interesting phenotypes, especially for metal-related traits.

\section{ENHANCED NUTRIENT ACCUMULATION AND USE EFFICIENCY}

A lot of progress to understand the molecular and physiological mechanisms that control specific steps of the nutrient homeostasis network has been done in the last years, leading to improved nutrient accumulation and use efficiency in crop plants. However, our basic knowledge on metal 
homeostasis is far from complete, and its understanding is an urgent step needed to sufficiently enhance plant mineral acquisition and storage (Ghandilyan et al., 2006).

Quantitative Trait Loci (QTL) analysis is a powerful approach to detect chromosomal regions affecting quantitative traits, and several QTL mapping have been conducted for rice grain nutrients accumulation. Using crosses of $O$. sativa $\times O$. rufipogon, Garcia-Oliveira et al. (2009) and Hu et al. (2016) were able to detect numerous QTLs for different mineral nutrients accumulation in rice grains, and wild rice (O. rufipogon) contributed with favorable alleles for most of the QTLs. It is important to highlight that both authors found co-locations of QTLs for some minerals, suggesting that the same allele could be used to simultaneously improve rice grains nutritional quality. These QTLs are good candidates for fine-mapping and cloning of candidate genes.

Studies comparing wild and cultivated rice responses to nutrient deficiencies or use efficiencies are scarce. Chiangmai and Yodmingkhwan (2010) compared the responses of wild and cultivated rice varieties to phosphate $(\mathrm{Pi})$ application, and found that $O$. rufipogon have greater response to $\mathrm{Pi}$-application than cultivated rice varieties. The positive aspect of $O$. rufipogon is that it may be used as germplasm for improving the cultivated rice varieties responses to P-application. On the other hand, the $\mathrm{F} 1$ hybrid of the $O$. rufipogon and cultivated rice varieties may be more adaptive to the environment and more competitive in the rice field, being more difficult to eradicate (Chiangmai and Yodmingkhwan, 2010). It would be interesting to test the interaction of two factors: nutrient application (in different concentrations) and rice genotypes, including cultivated and wild species. This would help to compare the response of rice varieties to nutrient application more effectively.

Cultivated rice (O. sativa) accumulates high concentration of silicon ( $\mathrm{Si}$ ), which is required for its high and sustainable production. Mitani-Ueno et al. (2014) showed that Si uptake was lower in O. rufipogon, O. barthii, O. australiensis, and O. punctata, compared to the cultivated rice, O. sativa. However, when grown in the field, all of them showed similar concentration of $\mathrm{Si}$ in the shoots. Therefore, Si uptake ability by the roots is different within wild rice species and cultivated rice, but the similar shoot $\mathrm{Si}$ concentration suggests that wild rice has an ability to optimize Si accumulation in the shoots, which is important for healthy plant growth and production (Mitani-Ueno et al., 2014). According to the authors, high Si accumulation in cultivated rice is achieved by a high expression of both influx (Lsi1) and efflux (Lsi2) Si transporters in roots. As wild rice species can optimize Si accumulation in the shoots, even with lower mRNA expression of Lsi1 and Lsi2 genes, it would be interesting to find other molecular players that control Si homeostasis in Siefficient wild rice species. Since Lsi1 and Lsi2 transporters are also involved in As transport, a toxic, non-essential element that is also efficiently absorbed into rice plants, including grains (Clemens and Ma, 2016), the use of distinct alleles for these transporters (with different expression levels or altered affinities) from wild relative might be helpful to fine-tune Si/As uptake and distribution.

\section{NATURAL VARIATION AND METAL TOLERANCE}

Aluminum $\left(\mathrm{Al}^{3+}\right)$ toxicity is a widespread problem for crops growing in acidic soils, which compose around $40 \%$ of the arable land (Uexkuill and Mutert, 1995). At low $\mathrm{pH}, \mathrm{Al}^{3+}$ becomes highly available, severely decreasing root growth and leading to nutritional deficiencies and drought. Plants show $\mathrm{Al}$ avoidance mechanisms, which involves organic acid secretion to the rhizosphere and $\mathrm{Al}^{3+}$ chelation; and $\mathrm{Al}$ tolerance, in which $\mathrm{Al}^{3+}$ is detoxified inside the root vacuoles, avoiding toxicity to the cell wall (Kochian et al., 2015).

Rice is a model for Al-tolerant crops, since it developed both avoidance and tolerance mechanisms. NRAT1 (Nramp aluminum transporter) is a unique plasma membrane-localized transporter through which $\mathrm{Al}^{3+}$ enters the root symplast, working in concert with vacuolar transporters to detoxify $\mathrm{Al}^{3+}$ into the vacuoles (Kochian et al., 2015). Interestingly, NRAT1 locus was recently implicated in natural variation of $\mathrm{Al}$ tolerance in rice: tolerant rice accessions showed higher expression of NRAT1 than sensitive ones, and some sensitive accessions carry missense mutations in NRAT1 coding sequence (Li et al., 2014).

Oryza rufipogon, the common wild rice, is known as an $\mathrm{Al}$ tolerance species, with higher tolerance than cultivated rice, possibly even stimulating root elongation depending on $\mathrm{Al}^{3+}$ concentration (Nguyen et al., 2003; Cao et al., 2011). Crosses of $O$. rufipogon $\times O$. sativa were used to map many QTLs for Al stress tolerance (Nguyen et al., 2003). Interestingly, the major QTL, which explained $24.9 \%$ of the variation, is located in chromosome 3. NRAT1, associated with Al tolerance in cultivated rice germplasm, sits on chromosome 2, indicating that the major QTL from O. rufipogon is not NRAT1, and thus it would be possible to pyramid these tolerance genes. Moreover, other wild relatives are virtually unexplored for their Al tolerance, although they can be found in soils with high $\mathrm{Al}^{3+}$ levels (Vianello Brondani et al., 2005).

Iron toxicity is another well-known problem for rice production, especially for lowland rice. Upland rice is grown under non-flooded, aerobic conditions, which keeps $\mathrm{Fe}$ in its oxidized form $\left(\mathrm{Fe}^{3+}\right)$ that binds to soil particles, with low availability for uptake. However, under flooded conditions, Fe becomes highly available as $\mathrm{Fe}^{2+}$, leading to $\mathrm{Fe}$ overload and consequent toxicity (Ricachenevsky et al., 2010). Although there are rice cultivars with relative Fe tolerance, there is still need for improvement. In Africa, up to $60 \%$ of the rice area in West African countries is affected by this stress, which results in yield losses of 50\% (Audebert and Fofana, 2009). Interestingly, the other cultivated species of rice, African rice O. glaberrima, showed superior tolerance to Fe toxicity when compared to $O$. sativa, and thus could potentially be used to breed Fe excess tolerance into $O$. sativa cultivars. In a large screen, several accessions of $O$. glaberrima from the AfricaRice gene bank where tested for grain yield in the field under control and $\mathrm{Fe}$ toxicity conditions, and compared to O. sativa controls. Three accessions were found to have higher yield than O. sativa cultivars under stress, but comparable yield in control condition, and thus could 
be used in breeding programs (Sikirou et al., in press). Another recent study found seven QTLs for Fe tolerance in crosses of $O$. glaberrima $\times O$. sativa, with $O$. glaberrima contributing with the tolerance alleles (Dufey et al., 2015).

\section{FUTURE PERSPECTIVES}

Rice domestication was performed with a narrow genetic diversity, and less than $20 \%$ of wild species diversity is present in cultivated rice (Palmgren et al., 2014). Therefore, searching for variability in wild rice has great potential for rice breeding, since it might have important genes for enhanced nutrient accumulation and metal tolerance, already lost in cultivated rice. Even close relatives such as O. rufipogon or O. glaberrima present useful traits that are not found within $O$. sativa diversity. Breeding varieties that have improved nutrient use efficiency and accumulation depends on large genetic variation for root uptake, root-to-shoot transport, xylem and phloem loading and unloading, mineral remobilization/compartmentalization, and grain accumulation. Also, minimum requirements for nutrients must be fulfilled while simultaneously avoiding their possible toxic effects, as well as other toxic elements (Ghandilyan et al., 2006). This is not an easy task, since many transporters are not specific, being able to transport different nutrients with different specificities, including toxic ones. Then, in future genetic modification projects, we need to know which specific genes should be targeted, and also emphasize the expression in specific tissues and stages, instead of using constitutive expression. Natural variation in transporter genes might indicate important residues for selectivity, and useful sequences might be further improved by site-directed mutagenesis (Menguer et al., 2013; Yan et al., 2016). At the same time, search for genotypes with low concentration of anti-nutrients such as phytate might be fruitful (Ghandilyan et al., 2006).

Several genes that encode proteins important to mineral homeostasis, including membrane transporters, storage proteins,

\section{REFERENCES}

Atwell, B. J., Wang, H., and Scafaro, A. P. (2014). Could abiotic stress tolerance in wild relatives of rice be used to improve Oryza sativa? Plant Sci. 215-216, 48-58. doi: 10.1016/j.plantsci.2013.10.007

Audebert, A., and Fofana, M. (2009). Rice yield gap due to iron toxicity in West Africa. J. Agron. Crop Sci. 195, 66-76. doi: 10.1111/j.1439-037X.2008.00339.x

Baxter, I., Ouzzani, M., Orcun, S., Kennedy, B., Jandhyala, S. S., and Salt, D. E. (2007). Purdue ionomics information management system. An integrated functional genomics platform. Plant Physiol. 143, 600-611. doi: 10.1104/pp.106.092528

Cao, Y., Lou, Y., Han, Y., Shi, J., Wang, Y., Wang, W., et al. (2011). Al toxicity leads to enhanced cell division and changed photosynthesis in Oryza rufipogon L. Mol. Biol. Rep. 38, 4839-4846. doi: 10.1007/s11033-010-0618-9

Chiangmai, P. N., and Yodmingkhwan, P. (2010). Common wild rice (Oryza rufipogon Griff.) and some tropical rice (Oryza sativa L.) varieties response to inorganic phosphorus application. Silpakorn Univ. Sci. Tech. J. 4, 36-46.

Clemens, C., and Ma, J. F. (2016). Toxic heavy metal and metalloid accumulation in crop plants and foods. Annu. Rev. Plant Biol. 67, 489-512. doi: 10.1146/annurev-arplant-043015-112301 metal ligands with different substrate specificities, receptors and regulatory proteins have been identified in rice (Sperotto et al., 2009; Ricachenevsky et al., 2015). The knowledge accumulated will be key for fast characterization of protein involved in uptake and transport of metals in wild relatives, using comparative transcriptomics and functional characterization of orthologous genes (Mitani-Ueno et al., 2014). QTL mapping of traits in wild relatives should be explored, and introgression of desirable traits by crossing with O. sativa is feasible (Jena, 1994; Collard and Mackill, 2008). Genome-wide association study (GWAS), which has been successfully used to map complex traits in rice (Kumar et al., 2015), has great potential for identifying the underlying genes causing natural variation in nutrient accumulation and metal tolerance (Norton et al., 2014; Huang and Salt, 2016; McCouch et al., 2016).

Yet, to identify the available genetic variation, extensive screening studies on wild rice species accessions is the first step. As wild rice species are distributed across several biomes worldwide (Atwell et al., 2014), different research groups need to join efforts to uncover potentially useful traits. In order to facilitate the access to wild rice material and information, databases on molecular and physiological characteristics should be developed, likely in association with seed distribution centers such as Genesys (https://www.genesys-pgr.org/). Already existing platforms such as the IonomicsHub (www.ionomicshub.org; Baxter et al., 2007) might be a useful starting point. Although the use of crop wild relatives is still in its beginnings, the few studies available clearly indicate that we should move forward to unlock the full potential of these species for enhanced nutrient accumulation and metal tolerance.

\section{AUTHOR CONTRIBUTIONS}

Both authors have made substantial, direct and intellectual contribution to the work, and approved it for publication.

Collard, B. C., and Mackill, D. J. (2008). Marker-assisted selection: an approach for precision plant breeding in the twenty-first century. Philos. Trans. R. Soc. Lond. B Biol. Sci. 363, 557-572. doi: 10.1098/rstb.2007.2170

Dufey, I., Draye, X., Lutts, S., Lorieux, M., Martinez, C., and Bertin, P. (2015). Novel QTLs in an interspecific backcross Oryza sativa $\times$ Oryza glaberrima for resistance to iron toxicity in rice. Euphytica 204, 609-625. doi: 10.1007/s10681014-1342-7

Elert, R. (2014). Rice by the numbers: a good grain. Nature 514, S50-S51. doi: $10.1038 / 514$ S50a

Garcia-Oliveira, A. L., Tan, L., Fu, Y., and Sun, C. (2009). Genetic identification of quantitative trait loci for contents of mineral nutrients in rice grain. J. Integr. Plant Biol. 51, 84-92. doi: 10.1111/j.1744-7909.2008.0 0730. $\mathrm{x}$

Ghandilyan, A., Vreugdenhil, D., and Aarts, M. G. M. (2006). Progress in the genetic understanding of plant iron and zinc nutrition. Physiol. Plant. 126, 407-417. doi: 10.1111/j.1399-3054.2006.00646.x

Hu, B. L., Huang, D. R., Xiao, Y. Q., Fan, Y. Y., Chen, D. Z., and Zhuang, J. Y. (2016). Mapping QTLs for mineral element contents in brown and milled rice using an Oryza sativa $\times$ O. rufipogon backcross inbred line population. Cereal Res. Commun. 44, 57-68. doi: 10.1556/0806.43.2015.044 
Huang, X. Y., and Salt, D. E. (2016). Plant Ionomics: from elemental profiling to environmental adaptation. Mol. Plant. 9, 787-797. doi: 10.1016/j.molp.2016.05.003

Jacquemin, J., Bhatia, D., Singh, K., and Wing, R. A. (2013). The International Oryza Map Alignment Project: development of a genus-wide comparative genomics platform to help solve the 9 billion-people question. Curr. Opin. Plant Biol. 16, 147-156. doi: 10.1016/j.pbi.2013.02.014

Jena, K. K. (1994). Production of intergeneric hybrid between Oryza sativa L. and Porteresia coarctata. Curr. Sci. 67, 9-10.

Kochian, L. V., Piñeros, M. A., Liu, J., and Magalhães, J. A. (2015). Plant adaptation to acid soils: the molecular basis for crop Aluminum resistance. Annu. Rev. Plant Biol. 66, 571-578. doi: 10.1146/annurev-arplant-043014-114822

Koutroubas, S. D., and Ntanos, D. A. (2003). Genotypic differences for grain yield and nitrogen utilization in Indica and Japonica rice under Mediterranean conditions. Field Crops Res. 83, 251-260. doi: 10.1016/S0378-4290(03)0 0067-4

Kumar, V., Singh, A., Mithra, S. V. A., Krishnamurthy, S. L., Parida, S. K., Jain, S., et al. (2015). Genome-wide association mapping of salinity tolerance in rice (Oryza sativa). DNA Res. 22, 133-145. doi: 10.1093/dnares/dsu046

Li, J. Y., Liu, J., Dong, D., Jia, X., McCouch, S. R., and Kochian, L. V. (2014). Natural variation underlies alterations in Nramp aluminum transporter (NRAT1) expression and function that play a key role in rice aluminum tolerance. Proc. Natl. Acad. Sci. U. S. A. 111, 6503-6508. doi: 10.1073/pnas.1318975111

Lu, F., Ammiraju, J. S., Sanyal, A., Zhang, S., Song, R., Chen, J., et al. (2009). Comparative sequence analysis of MONOCULM1-orthologous regions in 14 Oryza genomes. Proc. Natl. Acad. Sci. U. S. A. 106, 2071-2076. doi: 10.1073/pnas.0812798106

McCouch, S. R., Wright, M. H., Tung, C. W., Maron, L. G., McNally, K. L., and Fitzgerald, M. (2016). Open access resources for genome-wide association mapping in rice. Nat. Commun. 7, 10532. doi: 10.1038/ncomms10532

Menguer, P. K., Farthing, E., Peaston, K. A., Ricachenevsky, F. K., Fett, J. P., and Williams, L. E. (2013). Functional analysis of the rice vacuolar zinc transporter OsMTP1. J. Exp. Bot. 64, 2871-2883. doi: 10.1093/jxb/ert136

Mitani-Ueno, N., Ogai, H., Yamaji, N., and Ma, J. F. (2014). Physiological and molecular characterization of Si uptake in wild rice species. Physiol. Plant. 151, 200-207. doi: 10.1111/ppl.12125

Nguyen, B. D., Brar, D. S., Bui, B. C., Nguyen, T. V., Pham, L. N., and Nguyen, H. T. (2003). Identification and mapping of the QTL for aluminum tolerance introgressed from the new source, Oryza rufipogon Griff., into indica rice (Oryza sativa L.). Theor. Appl. Genet. 106, 583-593. doi: 10.1007/s00122-0021072-4

Norton, G. J., Douglas, A., Lahner, B., Yakubova, E., Guerinot, M. L., Pinson, S. R., et al. (2014). Genome wide association mapping of grain arsenic, copper, molybdenum and zinc in rice (Oryza sativa L.) grown at four international field sites. PLoS ONE 9:e89685. doi: 10.1371/journal.pone.0089685
Palmgren, M. G., Edenbrandt, A. K., Vedel, S. E., Andersen, M. M., Landes, X., Østerberg, J. T., et al. (2014). Are we ready for back-to-nature crop breeding? Trends Plant Sci. 20, 155-164. doi: 10.1016/j.tplants.2014.11.003

Ricachenevsky, F. K., Menguer, P. K., Sperotto, R. A., and Fett, J. P. (2015). Got to hide your $\mathrm{Zn}$ away: molecular control of $\mathrm{Zn}$ accumulation and biotechnological applications. Plant Sci. 236, 1-17. doi: 10.1016/j.plantsci.2015.03.009

Ricachenevsky, F. K., Sperotto, R. A., Menguer, P. K., and Fett, J. P. (2010). Identification of Fe-excess-induced genes in rice shoots reveals a WRKY transcription factor responsive to Fe, drought and senescence. Mol. Biol. Rep. 37, 3735-3745. doi: 10.1007/s11033-010-0027-0

Sikirou, M., Shittu, A., Konaté, K. A., Maji, A. T., Ngaujah, A. S., Sanni, K. A., et al. (in press). Screening African rice (Oryza glaberrima) for tolerance to abiotic stresses: I. Fe toxicity. Field Crops Res. doi: 10.1016/j.fcr.2016.04.016

Sperotto, R. A., Ricachenevsky, F. K., Duarte, G. L., Boff, T., Lopes, K. L., Sperb, E. R., et al. (2009). Identification of up-regulated genes in flag leaves during rice grain filling and characterization of OsNAC5, a new ABA-dependent transcription factor. Planta 230, 985-1002. doi: 10.1007/s00425-009-1000-9

Sperotto, R. A., Ricachenevsky, F. K., Waldow, V. A., and Fett, J. P. (2012). Iron biofortification in rice: it's a long way to the top. Plant Sci. 190, 24-39. doi: 10.1016/j.plantsci.2012.03.004

Uexkuill, H. R., and Mutert, E. (1995). Global extent, development and economic impact of acid soils. Plant Soil 171, 1-15. doi: 10.1007/BF00009558

Vianello Brondani, R. P., Zucchi, M. I., Brondani, C., Nakano Rangel, P. H., de Oliveira Borba, T. C., Rangel, P. N., et al. (2005). Genetic structure of wild rice Oryza glumaepatula populations in three Brazilian biomes using microsatellite markers. Genetica 125, 115-123. doi: 10.1007/s10709-005-4916-4

Yan, J., Wang, P., Wang, P., Yang, M., Lian, X., Tang, Z., et al. (2016). A lossof-function allele of OsHMA3 associated with high cadmium accumulation in shoots and grain of Japonica rice cultivars. Plant Cell Environ. doi: 10.1111/pce.12747. [Epub ahead of print].

Zhu, Q., Zheng, X., Luo, J., Gaut, B. S., and Ge, S. (2007). Multilocus analysis of nucleotide variation of Oryza sativa and its wild relatives: severe bottleneck during domestication of rice. Mol. Biol. Evol. 24, 875-888. doi: $10.1093 / \mathrm{molbev} / \mathrm{msm} 005$

Conflict of Interest Statement: The authors declare that the research was conducted in the absence of any commercial or financial relationships that could be construed as a potential conflict of interest.

Copyright (c) 2016 Ricachenevsky and Sperotto. This is an open-access article distributed under the terms of the Creative Commons Attribution License (CC BY). The use, distribution or reproduction in other forums is permitted, provided the original author(s) or licensor are credited and that the original publication in this journal is cited, in accordance with accepted academic practice. No use, distribution or reproduction is permitted which does not comply with these terms. 